\title{
VIEWPOINT
}

\section{The Athlete's Return in the Post-COVID-19}

José Antônio Caldas Teixeira, ${ }^{1,2(1)}$ Mateus Freitas Teixeira, ${ }^{1(0)}$ Pedro Soares Teixeira, ${ }^{1(0)}$ Juliana Grael Jorge ${ }^{1(0)}$

Universidade Federal Fluminense - Hospital Universitário Antônio Pedro, ${ }^{1}$ Niterói, RJ - Brazil

Fit Center, Niterói, ${ }^{2} R J$ - Brazil

The disease caused by Coronavirus 2019 (COVID-19) is associated with significant mortality and morbidity, including pulmonary and cardiac adverse sequelae. ${ }^{1-3}$ It began in December 2019 in the city of Wuhan, and quickly became a pandemic, declared by the World Health Organization (WHO) on March 11 of 2019.4 By early September 2020, more than 28 million patients have been confirmed infected by COVID-19 in more than 200 countries. $^{3}$

Infection by COVID-19 can develop with severe acute respiratory syndrome (SARS-CoV-2), acute respiratory distress syndrome (ARDS), neurological, cardiac and vascular manifestations being not unusual, and are associated with increased mortality. ${ }^{1,-6}$ The pattern of COVID-19 infection and its late sequelae has its pathogenesis only partially elucidated. ${ }^{1,5}$

Most individuals infected with COVID-19 will be either asymptomatic or will have mild to moderate symptoms ${ }^{1,5}$. The highest mortality rates are observed in patients over 60 years old and with comorbidities, especially cardiovascular diseases, obesity, and diabetes. Patients with worst outcomes also had high serum levels of ultra-sensitive troponin (Tus) and other inflammatory markers, such as interleukin 6 and ferritin. . $^{1-5,7}$

Notably, the lungs are the main organs involved by COVID-19, and angiotensin-converting enzyme 2 (ACE2) is part of the virus pathway into the lung cell. ACE2 is not only found in the lungs, but also in several other organs, including the heart, the endothelium of the cardiovascular system, the small intestine and

\section{Keywords}

COVID-19; Betacoronavirus; Pandemic; Athletes; Sport; Exercise; Return to Sport/psychology; Patient Discharge. the nervous system..$^{1,3,4,7}$ The inflammatory response described as "cytokine storm" seems to be responsible for ARDS, vascular/endothelial inflammation, myocarditis, and related events such as arrhythmias, ventricular dysfunction, and sudden death.

Based on Chinese data, cardiac injury appears to be a relevant feature of the disease, occurring in 20 to $30 \%$ of hospitalized patients and contributing to $40 \%$ of deaths. Cardiovascular complications have been described, such as: myocardial injury (20\%), arrhythmias (16\%), myocarditis (10\%), acute heart failure (HF) and shock (up to $5 \%$ of cases), sometimes with a dysfunction similar to Takotsubo's disease. These data may vary depending on the authors. ${ }^{1-5,7}$

Specific Complications in the Organs and Influences in Athletes

\section{Lungs}

Lungs are the organs most frequently involved by COVID-19. Pneumonia affects about $20 \%$ of those tested positive in China, $14 \%$ with severe or critical forms of pneumonia, characterized by pulmonary infiltration affecting more than $50 \%$ of the lungs with important hypoxia. ${ }^{1,5}$

During SARS outbreak in 2002-2003, affected patients showed pulmonary restriction, with reduced pulmonary diffusion capacity and functional capacity for at least 2 years of follow-up. ${ }^{1,5}$ Recently, in a case report from the University of Innsbruck, six divers also developed severe pulmonary changes after being affected by COVID-19. ${ }^{5}$

It is noteworthy that, in athletes, even small reductions in ventilatory capacity and pulmonary diffusion might affect performance. ${ }^{5}$ Exercise capacity would be limited due to changes in pulmonary diffusion, which may

Mailing Address: José Antônio Caldas Teixeira

Rua Marquês de Paraná 303, Centro. Postal Code: 24033-900, Niterói, RJ - Brazil.

E-mail: jacaldas_@hotmail.com 
trigger pulmonary arterial hypertension, right heart overload and, in the long-term, cor pulmonale., ${ }^{1,5}$

Nieß et al., ${ }^{5}$ recommend that spirometry should be performed on those athletes with a history of pulmonary involvement due to COVID-19 infection, as it may allow, and better elucidate functional changes. ${ }^{5}$ When available, preliminary findings in spirometry, performed during annual assessments, could be comparatively used to help analyze these sequelae.

Some authors recommend non-invasive capillary observation of arterial oxygen saturation, by pulse oximetry along with exercise test (ET) or cardiopulmonary exercise test (CPET). ${ }^{5}$ CPET associate's variables allow a better demonstration of a possible pulmonary limitation to exercise: respiratory equivalents, the slope of the VE/ $\mathrm{VCO}_{2}$ ratio, in addition to the drop in $\mathrm{SatO}_{2}$. In the case of identified alterations compatible with a pulmonary limitation, we should deepen this functional assessment by testing the diffusing capacity of the lungs for carbon monoxide (DLCO) and possible imaging exams. ${ }^{5}$

\section{Cardiovascular System}

Recognizing a possible myocardial involvement during COVID-19 infection is of cardinal importance for athletes. Myocarditis is one of the main causes of sudden death in athletes under 35 years old, ${ }^{8,9}$ with viral myocarditis emerging after viruses with respiratory and/ or gastrointestinal involvement. ${ }^{6,8,9}$
Of patients with the severe form of COVID-19, 15\% to $31 \%$, had an increase in Tus during hospitalization, which could indicate cardiac involvement and myocardial injury, ${ }^{1-4,7}$ and might be related to their prognosis. ${ }^{3,4}$

Cardiac involvement is demonstrated by increased Tus levels, electrocardiographic (ECG) and echocardiographic (ECO) changes, mentioned in more than $22 \%$ of hospitalized patients with COVID-19, compared to $1 \%$ of patients with other viruses. ${ }^{10}$

Different Factors are Related to Myocardial Damage and Can be Dummarized in Chart 1 . $^{7}$

Cardiac involvement is best demonstrated by cardiac nuclear magnetic resonance (MRI), which allows the identification of edema, fibrosis and contractile dysfunction. ${ }^{1,3,4,7}$ The question that remains open is whether there is a risk of myocardial involvement in mild and moderate forms or even in asymptomatic. ${ }^{5,11} \mathrm{Nie} ß$ et al. mention the occurrence of severe myocarditis in the convalescence phase as well as cases of sudden death in patients infected by COVID-19 with home treatment. ${ }^{5}$

\section{The Athlete Facing the Pandemic}

Engaging in some type of physical activity is an important component of a health promotion program and is recommended to be done by all those who are isolated at home. ${ }^{11,13}$

\section{PROPOSED HEART INJURY MECHANISMS IN COVID-19}

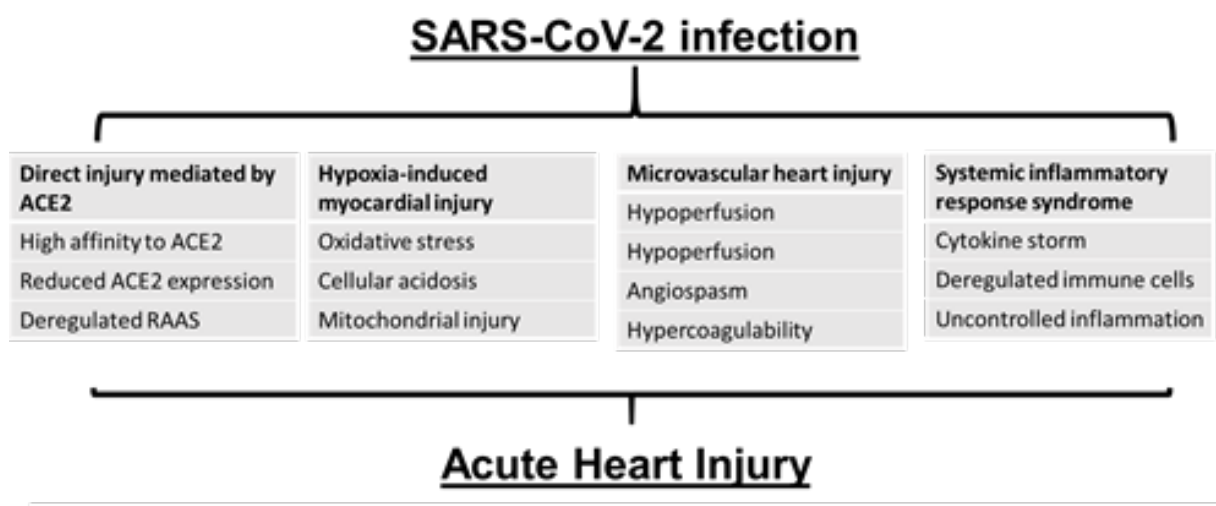

ACE2 - angiotensin-converting enzyme receptor 2; Covid-19- name of the disease caused by coronavirus 2; SARS-COV2

- Severe Acute Respiratory Syndrome Coronavirus 2; RAAS - renin angiotensin aldosterone system. 
There is no specific data available on prevalence, nature and behavior of the disease associated with COVID-19 in the athlete. ${ }^{5,6,11}$ The increasing reports about pulmonary and cardiac involvement, in mild to moderate clinical forms ${ }^{2}$, points out the need for a new health policy in relation to athletes, for both recreational and professionals sports. A new challenge arises in determining when and how the athletes infected with COVID-19, especially those who had the disease and recovered, will have medical clearance to return to training and competing. $2,5,6,10,11$

Existing epidemiological and clinical data are scarce to safely guide this process. Notably, the numbers of asymptomatic cases in the community, the prevalence of cardiac injury in non-hospitalized individuals and the long-term evolution of those who have had proven cardiac involvement, all of these questions are still unanswered. ${ }^{10}$

In this pandemic, the athlete theme, with its specific particularities, is pertinent. It includes concerns about continuing training, risk of transmission between team members, and the potential deleterious effect of vigorous exercise increasing susceptibility to COVID-19 infection. ${ }^{10,11,13}$

Intense training, with or without a sudden increase in intensity, are associated with transient immune changes, elevation of systemic inflammatory markers, oxidative stress and muscle damage. ${ }^{11}$ A potential increase in the risk of falling ill during periods of high training load is a reality $5,6,11,13$. The transmission of COVID-19 is facilitated in several training scenarios, such as: in group sports, contact sports, not obeying the distancing rule, sharing equipment, not following universal rules of personal hygiene, in addition to the use of collective changing rooms.

Athletes often present with non-specific symptoms, like fatigue, asthenia, reduced performance, myalgia and disproportionate tachycardia at rest. The last might be blurred by a mix of differential diagnosis in this population such as overtraining syndrome, anxiety and depression. In such way, the diagnosis of an infection, or even cardiac involvement due to COVID-19, will not be suspected. Thus, additional diagnostic and therapeutic measures might be delayed and neglected. ${ }^{6}$

COVID-19 cardiac injury can have long-term repercussions, and we can underestimate it if we only define it by the increase in Tus. In those athletes with mild symptoms, cardiac injury should always be ruled out, and even in those with abnormal baseline exams, the diagnosis cannot be given with certainty. Some of the changes in the ECG, for example, may be the consequences of adaptations to prolonged training, as well as transient elevations of creatine phosphokinase and Tus, which may occur due to the acute effect of a high-volume session or training intensity. ${ }^{6}$

Another observation is that the typical presentation of a suspected myocarditis differs from the scenario observed in a COVID-19 hospitalized patient compare to an athlete with mild symptoms of the disease. Athletes are extremely sensitive to changes in their health status with reduced performance. Attention must be given to prolonged recovery time to training stimuli, dyspnea and/or fatigue disproportionate to the effort performed, as well as greater myalgia triggered by training, all may represent unspecific symptoms of COVID-19 infection. ${ }^{6}$

Possible pulmonary sequelae, even if without clinical significance for everyday life, can be highly relevant for those who participate in competitive sports, which imposes high training loads, and cannot rule out damaging effects that may occur in their performance. The prolonged convalescence process itself with persistent symptoms of fatigue, asthenia, myalgia certainly decreases physical performance after an infection by COVID-19.5,10,11

\section{Recommendations to Return to Training}

The recommendations to return to training after an airway infection were that those with signs and symptoms of infection restricted to the upper airways (nasal congestion, runny nose), should not be removed from training ${ }^{11}$. The scientific evidence for this recommendation was weak, and the potential risk of developing complications as myocardial damage due to vigorous training was known.

Currently, for COVID-19 viruses, recommendation for the return to training, even with only upper airway symptoms, is to prolong the period of absence and use more conservative return strategies (10 to 14 days after symptom onset plus 7 to 14 days of symptom absence). ${ }^{5,10,11}$

Due to the great variability in the severity of Covif-19 infection, variation in the scope of evaluation also seems logical in the evaluation to return to play a competitive sport with the intensity of training that it usually requires. ${ }^{2,5,6,10,11}$

Some societies and institutions (American College of Cardiology's Sports \& Exercise Cardiology Council, 
International Olympic Committee) started to provide guidance, based on expert opinions, regarding the return of athletes to competitive practice. $5,6,10,11$

\section{Recommendations for Screening Athletes in Post- COVID-19}

The categorizations are based on the clinical signs and symptoms obtained initially through an anamnesis and physical exam, associated with the clinical findings on pulmonary, cardiovascular and neuromuscular system involvement, in addition to the results of the screening tests. Recommendations based on algorithms must be individualized and adapted with respect to the individual course of the disease, the use or not of therapies, such as hydroxychloroquine that prolongs QTc interval, and additional diagnostic measures required. $2,5,6,10,11$

The definition of the onset of typical symptoms of the disease must be performed to estimate the time of medical clearance to return training, in addition to basic and specific laboratory tests, these depending on the involvement of each case.

Nieß et al. ${ }^{5}$ proposed a categorization of patients shown in Table 1. These recommendations may be valid for today and only for a short period in the light of the development of new knowledge, and may need to be reviewed. ${ }^{5,6,10,11}$

\section{Our Recommendation}

After considering the recommendations for evaluating athletes from different authors, $2,5,6,10,11$ we make the following suggestions.

All athletes returning to training and presenting to the medical department, must undergo a clinical history and a physical examination, highlighting the search for compatible signs and symptoms of COVID-19 infection. In case of a positive history, characterize the onset of symptoms, its evolution, tests performed, hospitalization and when asymptomatic. This way, we can see ourselves in different situations (Chart 2).

\section{I - Asymptomatic and negative tests}

Asymptomatic athletes, with no reported disease. In addition to anamnesis and physical examination, routine biochemical tests, and baseline ECG. All athletes should be tested for antigen and antibodies for COVID-19. If all negative for COVID-19 (RT-

Table 1 - Proposed categorization for COVID-19 patients.

\section{Categorization}

A - Positive tests for COVID-19, but no signs and symptoms of infection

B - Positive tests and patient with signs and symptoms of the disease, such as: fever with temperature above 37.8 , dry cough, myalgia, headache, loss of smell and/or taste etc., but without confirmed pneumonia

C - COVID-19 infection with confirmed pneumonia

D - COVID-19 infection with suspected or confirmed myocarditis with or without pulmonary involvement and with or without other symptoms

History and Physical Examination

Assess the severity of the evolution of the disease, symptoms related to exercise, such as: chest pain, cough and/or dyspnea (in each case especially if induced by exercise), fever and its duration, dizziness, myalgia, fatigue, headache, loss of taste and smell, mood changes, medications used and your sports background

Highlight in the evaluation of the affected lymph nodes and chains, baseline heart rate, blood pressure in different positions, pulmonary examination (see wheezing and crackles), peripheral and cardiac cardiovascular examination (see arrhythmias, accessory sounds, murmurs and attritions), abdomen (with emphasis on visceromegaly), body temperature and basic neurological examination (assessing neuropathies).

Laboratory analysis

Complete blood count, US C-Reactive Protein (PCR), transaminases, creatine phosphokinase (CPK), urea, creatinine, blood glucose, urinalysis.

Depending on the clinical history data, physical examination and the above results: ferritin, troponin, D-dimer, interleukin 6, procalcitonin, nasal swab and oropharynx RT-PCR, antibody search for COVID-19 (IgM, IgA, IgG)

Nieß et al . 


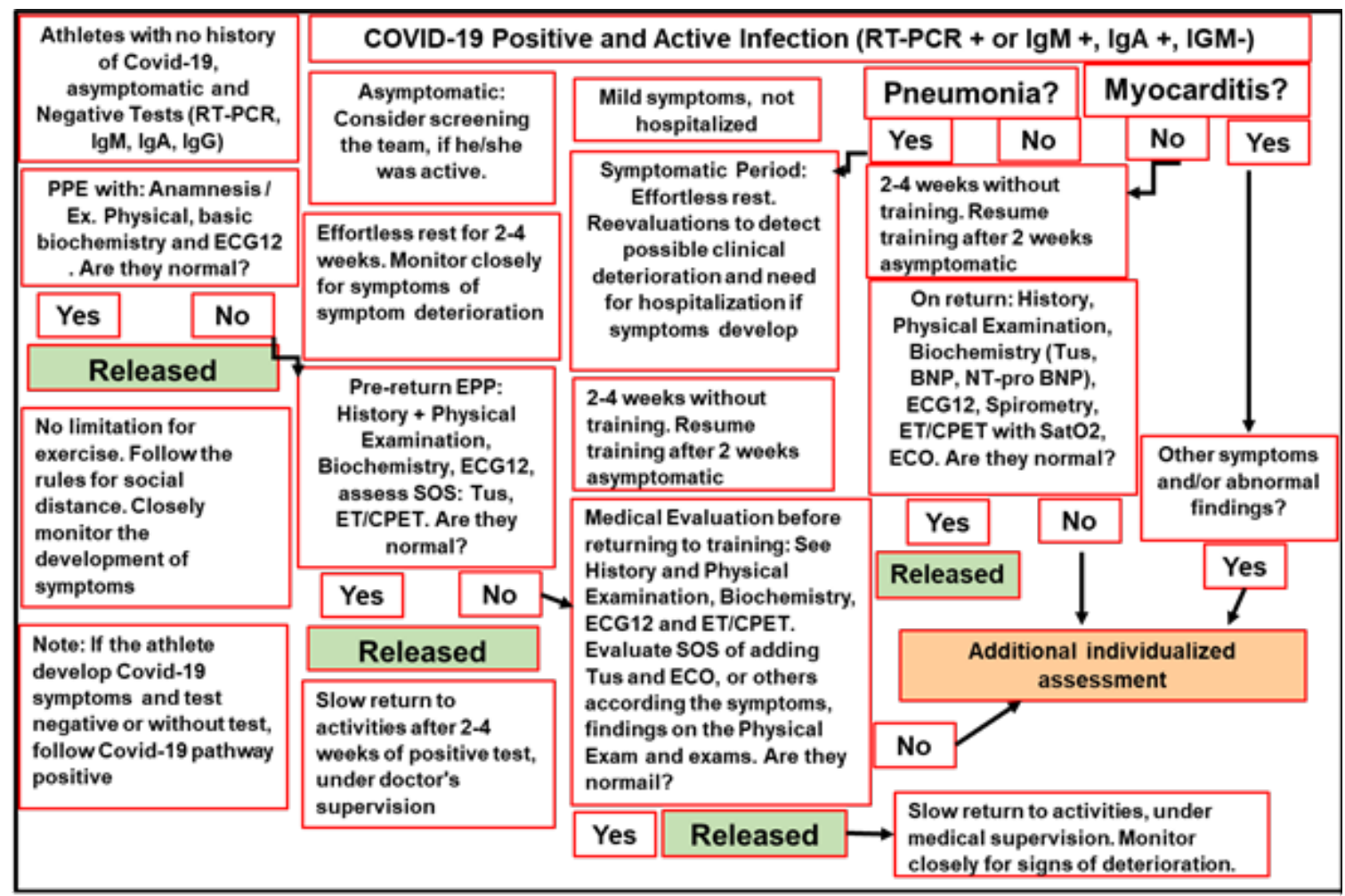

Legends: Covid-19 - name of the disease caused by coronavirus 2: PPE - pre participation evaluation; RT-PCR - reverse-transcriptase polymerase chain reaction; ECG - electrocardiogram; Ex. - exam; ET - exercise test; CPET - cardiopulmonary stress test; Tus - ultra sensitive troponin; SOS - if necessary; ECO - echocardiogram; Ig - immunoglobulin; BNP - type B natriuretic peptide; NT-pro BNP - Inactive N-terminal of the pro-BNP.

Figure 2 - Recommended evaluation flowchart for post - COVID-19 in athletes. Adapted from Schellhorn et al. ${ }^{6}$

PCR-, IgM-, IgA-, IgG-), the athlete will be allowed to return to training, without additional specific evaluation. ${ }^{10}$

II - Asymptomatic with positive tests

II a) Asymptomatic with positive tests for active infection Asymptomatic patients, but with positive tests showing active infection (RT-PCR+, IgM+, IgA+, IgG-), must stay at home following the strict rules of home isolation and without training, for at least two weeks. The duration of the disease is shown to be critical to the patient's clinical outcome, with an increased risk of deterioration after the first week of symptom onset. Therefore, it is recommended to follow closely after the onset of the first symptoms and to establish marks in this evolution and recommendations.

After two weeks of observation, if they remain asymptomatic, they should be reassessed by the medical department for prior evaluation, in addition to have their clinical evolution monitored for another two weeks, plus another evaluation before slowly returning to training. ${ }^{10}$

This group of athletes represents, by the current data, a great unknown. Due to reports of cardiac and pulmonary involvement, even in asymptomatic patients, our opinion, and agreeing with some reviewed authors, ${ }^{5,10}$ is that, in addition to the anamnesis, physical examination, blood routine, resting ECG, Tus and an ET/CPET should be added, with additional investigation if in the presence of altered exams.

II b) Asymptomatic athletes, but positive tests for previous infection

In asymptomatic athletes who detect previous infection, only with IgG + and the other negative tests (RT-PCR-, IgM-, IgA-, IgG +), an assessment like those in group II a who are returning after the isolation period is recommended. In these 
two populations, the clinical examination, ECG, and additional stress tests (ET/CPET), should be performed, especially if they demonstrate clinical signs of cardiopulmonary symptoms and/or changes in the resting ECG and stress tests. ${ }^{10}$

III - Athletes who had symptomatic disease and positive test for previous disease

In those symptomatic athletes and tested positive for previous disease (RT-PCR-, IgM-, IgA-, IgG+), with or without pulmonary involvement, but without the severe form of the disease, in the pre-return assessment, in addition to clinical history, physical examination and ECG, routine biochemical and an ET or, preferably, a CPET associated with $\mathrm{SatO}_{2}$. Depending on the clinical findings and the results of these tests, associate biochemical markers of injury and cardiac dysfunction (Tus, natriuretic peptide type $\mathrm{B}(\mathrm{BNP})$, inactive $\mathrm{N}$-terminal proBNP (NT-proBNP)) and an echocardiogram (ECO). We also recommend a baseline control spirometry and a 24h Holter. . $^{50}$

Athletes with a history of illness in the most severe forms portray a high-risk population. Although we know that myocarditis is not present in the majority of patients hospitalized by COVID-19, it is recommended to evaluate these patients thinking as if they had had it, and to follow the recommendations for complete evaluation for myocarditis in the pre-participation evaluation. ${ }^{10}$

If the biochemical markers of injury and dysfunction (Tus, BNP, NT-proBNP), ECG and ECO are normal, it is recommended at least 2 to 4 weeks after the symptoms cease of relative rest. They will then undergo a careful cardiac clinical reassessment, prior examinations and a CPET should be repeated before resuming gradual training. ${ }^{8-10}$

If evidence of myocardial involvement is found, along with high levels of Tus, BNP, NT-proBNP and complemented by the findings on physical examination, an MRI should be performed. ${ }^{3,5}$

IV) Suspected and/or confirmed cases of myocarditis

In a suspected and/or confirmed case of myocarditis, we must follow the existing guidelines in relation to this entity while providing full medical clearance to return to sport. ${ }^{5,8,9}$

Myocarditis due to viral aggression can lead to dysfunction, arrhythmias, and death. During the acute phase, the practice of exercise can result in accelerating viral replication, increasing the inflammatory component and cellular necrosis, and providing a pro arrhythmic substrate. ${ }^{10}$

Returning to sports after myocarditis is based on the normalization of inflammation and dysfunction biomarkers, myocardial function, and absence of resting and/or stress-induced arrhythmias. ${ }^{8-10}$ A new risk stratification will take place from 3 to 6 months of withdrawal from their sports activities according to the clinical follow-up and complementary exams (biochemical markers of injury and myocardial dysfunction, ECO, ET/CPET, 24h Holter and MRI). $5,6,8-10$

The prognosis, for myocarditis for other viruses, is generally favorable in those with acute myocarditis, with normalization of ventricular function, and absence of fibrosis areas on MRI, however, we still do not know the long-term prognosis of COVID-19 myocarditis. We must keep in mind that athletes who developed cardiovascular complications due to COVID-19 infection may have late manifestations, e.g., arrhythmias, and deserve to have a long-term follow-up.

Data on athletes who have recovered from COVID-19 infection will be of the utmost importance. To optimize the counseling and treatment of future cases, prospective data should be collected in this current pandemic.

\section{Author Contributions}

Conception and design of the research: Teixeira JAC. Acquisition of data: Teixeira JAC, Teixeira MF, Teixeira PS, Jorge JG. Analysis and interpretation of the data: Teixeira JAC. Writing of the manuscript: Teixeira JAC, Teixeira MF, Teixeira PS, Jorge JG.

\section{Potential Conflict of Interest}

No potential conflict of interest relevant to this article was reported.

\section{Sources of Funding}

There were no external funding sources for this study.

\section{Study Association}

This study is not associated with any thesis or dissertation work. 


\section{References}

1. Barker-Davies RM, O'Sullivan O, Senaratne KPP, Baker P, Cranley M Dharm-Datta $S$, et al. The Stanford Hall consensus statement for postCOVID-19 rehabilitation. Br J Sports Med. 2020;54(16):949-59

2. Dores, H \& Cardim N. Return to play after COVID-19: a sport cardiologist's view. Br J Sports Med Month. 2020;54(19):1132-3.

3. Huang L, Zhao P, Tang D, Zhu T, Han R, Zhan C, et al. Cardiac involvement in recovered COVID-19 patients identified by magnetic resonance imaging, JACC: Cardiovasc Imaging.2020;13(11):2330-9.

4. Costa IBSS, Bittar CS, Rizk SI, Araujo Filho AE, Santos KAQ, Machado TIV, et al. The Heart and COVID-19: What Cardiologists Need to Know. Arq Bras Cardiol. 2020; 114(5):805-16

5. Nieß AM, Bloch W, Friedmann-Bette B, Grim C, Halle M, Hirschmüller A, et al. Position stand: return to sport in the current Coronavirus pandemic (SARS-CoV-2 / COVID-19). Dtsch Z Sportmed. 2020; 71: E1-E4.

6. Schellhorn P, Klingel K, Burgstahler C. Return to sports after COVID-19 infection. Do we have to worry about myocarditis? Eur Heart J 2020;41(46):4382-4.

7. Brandão, SCS; Andrade, AW; Feitosa, ADM. COVID-19 e Coração. Manual Prático de Condutas. Abril,2020. ISBN: 978-65-00-020663Doi:10.00011/jamacardiol.2020.2136 Epub ahead print
8. Ghorayeb N, Stein R, Daher DJ, Silveira AD, Ritt LEF, Santos DFP, et al. Atualização da Diretriz em Cardiologia do Esporte e do Exercício da Sociedade Brasileira de Cardiologia e da Sociedade Brasileira de Medicina do Esporte - 2019. Arq Bras Cardiol. 2019; 112(3):326-68.

9. Maron BJ, Udelson JE, Bonow RO, Nishimura RA, Ackerman MJ, Mark Estes III NA, et al. Eligibility and Disqualification Recommendations for Competitive Athletes with Cardiovascular Abnormalities: Task Force 3: Hypertrophic Cardiomyopathy, Arrhythmogenic Right Ventricular Cardiomyopathy and Other Cardiomyopathies, and Myocarditis A Scientific Statement from the American Heart Association and American College of Cardiology. J Am Coll Cardiol.2015;66(21):2362-71.

10. Phelan D, Kim JH, Chung EH. A Game Plan for the Resumption of Sport and Exercise After Coronavirus Disease 2019 (COVID-19) Infection. JAMA ,2020; doi: 10.0001/jamacardiol..2020.2136 Epub ahead print

11. Hull JH, Loosemore M, Schwellnus M. Respiratory health in athletes: facing the COVID-19 challenge. Lancet Respir Med. 2020;8(6):557-8.

12. Zubair AS, McAlpine LS, Gardin T, Farhadian S, Kuruvilla DE, Spudich S. Neuropathogenesis and Neurologic Manifestations of the Coronaviruses in the Age of Coronavirus Disease 2019: A Review. JAMA Neurol.2020;77(8):1018-27.

13. Castro RRT, Silveira Neto JG, Castro RRT. Exercise Training: A Hero that Can Fight two Pandemics at Once.Int J Cardiovasc Sci.. 2020;33(3):284-7. 